\title{
Decomposing graphs into a spanning tree, an even graph, and a star forest
}

\author{
Kasper Szabo Lyngsie Martin Merker* \\ Department of Applied Mathematics and Computer Science \\ Technical University of Denmark \\ Lyngby, Denmark \\ $\{\mathrm{ksly}$, marmer\}@dtu.dk
}

Submitted: Jun 29, 2018; Accepted: Feb 21, 2019; Published: Mar 8, 2019

(C) The authors. Released under the CC BY-ND license (International 4.0).

\begin{abstract}
We prove that every connected graph can be edge-decomposed into a spanning tree, an even graph, and a star forest.
\end{abstract}

Mathematics Subject Classifications: 05C05, 05C38

\section{Introduction}

All graphs in this paper are simple and finite. A decomposition of a graph $G$ is a collection of edge-disjoint subgraphs whose union is $G$. A graph is called even if every vertex has even degree. It is easy to see that every graph can be decomposed into a forest and an even graph. In 1979, Malkevitch [9] studied cubic graphs which admit such a decomposition where the forest is a spanning tree. In this case it is equivalent to the existence of a spanning tree containing no vertices of degree 2. Such a spanning tree is called homeomorphically irreducible, or a HIST.

For general graphs, the existence of a HIST is much less restrictive than the existence of a decomposition into a spanning tree and an even graph. However, even the existence of a HIST is not guaranteed by large connectivity or regularity, as was shown by Albertson et al. [3]. Douglas [5] showed that it is NP-complete to decide whether a planar subcubic graph contains a HIST.

In cubic graphs, the removal of the edges of a spanning tree results in a collection of cycles and paths. Hoffmann-Ostenhof [4] (see also [6]) conjectured that the spanning tree can be chosen such that the collection of paths is a matching.

\footnotetext{
*Supported by the Danish Council for Independent Research, Natural Sciences, grant DFF-1323-00178, AlgoDisc.
} 
Conjecture 1 (3-Decomposition Conjecture). Every connected cubic graph can be decomposed into a spanning tree, a collection of cycles, and a matching.

Akbari, Jensen, and Siggers [2] showed that any cubic graph has a decomposition into a spanning forest, a collection of cycles, and a matching. Abdolhosseini et al. [1] verified the 3-Decomposition Conjecture for traceable cubic graphs and Ozeki and Ye [10] verified it for 3-connected planar cubic graphs. The latter was extended by Hoffmann-Ostenhof, Kaiser, and Ozeki [7] to all planar cubic graphs. The following theorem is the main result of our paper and is in some sense a generalization of the 3-Decomposition Conjecture to the class of all connected graphs.

Theorem 2. Every connected graph can be decomposed into a spanning tree, an even subgraph, and a star forest.

As a special case our result implies that every cubic graph has a decomposition into a spanning tree, a collection of cycles, and a collection of paths of length at most 2. This was previously shown by Li and Cui [8].

One might be tempted to think that every connected graph admits a decomposition into a spanning tree, an even graph, and a matching. However, this is easily seen to be false since the complete bipartite graph $K_{2, n}$ has no such decomposition. Such a decomposition is also not guaranteed if we restrict our attention to regular graphs.

Theorem 3. For each $r \geqslant 4$, there exists an r-regular connected graph which has no decomposition into a spanning tree, an even graph, and a matching.

Proof. Let $r \geqslant 4$ be given and let $G$ be the graph obtained from $K_{r+1}$ by subdividing each edge once. Let $G^{\prime}$ be a graph obtained from $K_{r+1}$ by subdividing $r-2$ edges once and adding an edge between each pair of vertices of degree 2. For each vertex $v$ of degree 2 in $G$, let $G_{v}$ denote a copy of $G^{\prime}$. Now let $G^{\prime \prime}$ be obtained from the disjoint union of $G$ and all the graphs $G_{v}$ by adding edges between $v$ and the vertices of degree $r-1$ in $G_{v}$, for each vertex $v$ of degree 2 in $G$. Note that $G^{\prime \prime}$ is $r$-regular and any decomposition of $G^{\prime \prime}$ into a spanning tree, an even graph, and a matching also induces such a decomposition of $G$. Clearly, the even graph cannot contain any edges of $G$, therefore this corresponds to a decomposition of $G$ into a spanning tree and a matching. The graph $G$ has $r(r+1)$ edges, and every spanning tree of $G$ has $r+\frac{r(r+1)}{2}$ edges, thus the matching has to contain at least $\frac{r(r-1)}{2} \geqslant r+2$ edges. However, the size of a maximal matching in $G$ is $r+1$, so $G$ cannot be decomposed into a spanning tree and a matching.

The construction in the proof above shows that for $r$-regular graphs the size of the stars in the forest in Theorem 2 grows at least linearly in $r$.

\section{Proof of Theorem 2}

Before we begin the proof of our main theorem, we introduce a few definitions. 
Definition 4 (separating cycle). A cycle $C$ in a connected graph $G$ is called separating if $G-E(C)$ is disconnected.

Note that in the literature a cycle is called separating in a graph if the removal of its vertex-set results in a disconnected graph, while for us the removal of its edge-set is relevant. In particular, every cycle containing a vertex of degree 2 is separating.

Definition 5 (fragile). A graph $G$ is called fragile, if $G$ is connected and every cycle of $G$ is separating.

Fragile graphs have also been investigated in the context of planar graphs by HoffmannOstenhof et al. [7]. Their 2-Decomposition Conjecture, which is equivalent to Conjecture 1, states that every subcubic fragile graph can be decomposed into a spanning tree and a matching.

Note that if we remove an even subgraph $H$ such that $G-E(H)$ is connected and if we choose such a subgraph of maximal size, then $G-E(H)$ is fragile. In particular, every connected graph decomposes into an even graph and a fragile graph. Therefore it is sufficient to prove that every fragile graph has a decomposition into a spanning tree and a star forest. For brevity, we introduce the following notation.

Definition 6 (starlit). A spanning tree $T$ of a graph $G$ is called starlit if $G-E(T)$ is a star forest.

All we need to show is that every fragile graph contains a starlit spanning tree. We prove an even stronger result, where we prescribe that all edges at a specified vertex belong to the spanning tree in the decomposition.

Definition 7 ( $\boldsymbol{v}$-full). A spanning tree $T$ of a graph $G$ is called $\boldsymbol{v}$-full for some vertex $v$ in $G$, if all edges incident with $v$ in $G$ are also in $T$.

We can now state the theorem we are going to prove.

Theorem 8. If $v$ is a vertex in a fragile graph $G$, then $G$ has a starlit $v$-full spanning tree.

As already discussed, Theorem 2 follows immediately from Theorem 8 . We finish this section by proving Theorem 8 .

Proof of Theorem 8. Let $G$ be a counterexample of minimal size.

Claim 1: $G$ is 2-connected.

Proof. Suppose the claim is false and $u$ is a cutvertex in $G$. Let $K$ be a component of $G-u$, let $G_{1}$ denote the subgraph of $G$ induced by $V(K) \cup\{u\}$, and let $G_{2}$ denote the graph induced by the edges in $G-E\left(G_{1}\right)$. We can assume that $v \in V\left(G_{1}\right)$. Clearly $G_{1}$ and $G_{2}$ are fragile and contain fewer edges than $G$, so $G_{1}$ contains a starlit $v$-full spanning tree $T_{1}$, and $G_{2}$ contains a starlit $u$-full spanning tree $T_{2}$. Now the union of $T_{1}$ and $T_{2}$ is a starlit $v$-full spanning tree in $G$. 
Note that Claim 1 implies that the minimum degree of $G$ is at least 2 .

Claim 2: There are no adjacent vertices of degree 2 in $G$.

Proof. Suppose $x$ and $y$ are two adjacent vertices of degree 2 and let $z$ denote the neighbour of $y$ different from $x$. We may assume without loss of generality that $v \neq y$. The graph $G^{\prime}=G-x y$ is fragile, so by minimality of $G$ there exists a starlit $v$-full spanning tree $T^{\prime}$ of $G^{\prime}$. If $v \neq x$, then $T=T^{\prime}$ is also a starlit $v$-full spanning tree of $G$. If $v=x$, then we choose instead a starlit $z$-full spanning tree $T^{\prime \prime}$ of $G^{\prime}$. Now $T^{\prime \prime}+x y-y z$ is a starlit $v$-full spanning tree of $G$.

Let $H$ be the subgraph of $G$ induced by the vertices of degree at least 3 .

Claim 3: $H$ contains no isolated vertices and no cycles of length 3.

Proof. Suppose $u$ is an isolated vertex in $H$. That is, $u$ is a vertex of degree at least 3 in $G$ all of whose neighbours have degree 2 .

First, suppose $u=v$. Let $x$ be a neighbour of $u$, and $y$ the neighbour of $x$ different from $u$. By Claim 2, $y$ has degree at least 3 and is therefore not adjacent to $u$. Let $G^{\prime}$ be the graph obtained from $G$ by removing $x$ and adding the edge $u y$. Since $u$ has only one neighbour of degree greater than 2 in $G^{\prime}$, every cycle through $u$ is still separating. Thus, $G^{\prime}$ is fragile and contains a starlit $u$-full spanning tree $T^{\prime}$. Now $T=T^{\prime}-u y+u x+x y$ is a starlit $u$-full spanning tree of $G$.

Thus we can assume $u \neq v$. The graph $G^{\prime}=G-u$ is connected by Claim 1 . Clearly $G^{\prime}$ is fragile and therefore contains a $v$-full starlit spanning tree $T^{\prime}$. If $v$ is a neighbour of $u$, then $T=T^{\prime}+u v$ is a starlit $v$-full spanning tree of $G$. If $v$ is not a neighbour of $u$, then adding an arbitrary edge incident with $u$ to $T^{\prime}$ results in a starlit $v$-full spanning tree of $G$. This contradiction shows that the minimum degree of $H$ is at least 1 .

Finally, suppose $H$ contains a cycle $C$ of length 3 . Since every vertex of $C$ has degree at least 3, and since $G$ is 2-connected, it is easy to see that $C$ is not separating, which contradicts $G$ being fragile.

For $u \in V(H)$, we write $d_{H}(u)$ to denote the degree of $u$ in $H$ and $d_{G}(u)$ for its degree in $G$.

Claim 4: If $u$ is a vertex in $H$ different from $v$, then $d_{H}(u) \geqslant 2$.

Proof. Suppose $u$ is a vertex of degree 1 in $H, u \neq v$, and $x$ is the neighbour of $u$ in $H$. Let $G^{\prime}=G-u$. First, suppose that $v$ is not a degree 2 vertex adjacent to $u$ in $G$. By Claim 1, the graph $G^{\prime}$ is fragile, so it has a starlit $v$-full spanning tree $T^{\prime}$ by minimality of $G$. Now $T=T^{\prime}+u x$ is a $v$-full starlit spanning tree of $G$. Thus, we can assume that $v$ has degree 2 and is a neighbour of $u$ in $G$. Let $T^{\prime \prime}$ be a starlit $x$-full spanning tree of $G^{\prime}$. Clearly $T=T^{\prime \prime}+u v$ is a $v$-full spanning tree of $G$. Since $T^{\prime \prime}$ is $x$-full, the spanning tree $T$ is also starlit, contradicting our choice of $G$. 
Claim 4 implies that there exists a cycle in $H$. The following claim shows that there are at most two vertices in $H$ which have degree less than 3 in $H$.

Claim 5: If $u \in V(H)$ and $d_{H}(u)=2$, then either $u=v$ or $d_{G}(v)=2$ and $u v \in E(G)$.

Proof. Suppose $u$ is a vertex of degree 2 in $H, u \neq v$, and $d_{G}(v) \geqslant 3$ or $u v \notin E(G)$. Let $x$ and $y$ denote the neighbours of $u$ in $H$. Note that all other neighbours of $u$ in $G$ have degree 2. Let $G^{\prime}$ be the graph obtained from $G-u$ by adding the edge $x y$. Claim 1 implies that $G$ is connected and Claim 3 implies that $G^{\prime}$ has no multiple edges. For a cycle $C^{\prime}$ in $G^{\prime}$ containing $x y$, the corresponding cycle $C$ in $G$, which is obtained from $C^{\prime}$ by replacing $x y$ with the path $x u y$, is separating if and only if $C^{\prime}$ is separating. Thus, $G^{\prime}$ is fragile and contains a starlit $v$-full spanning tree $T^{\prime}$. If $x y \in E\left(T^{\prime}\right)$, then $T=T^{\prime}-x y+u x+u y$ is a starlit $v$-full spanning tree in $G$. Thus, we can assume $x y \notin E\left(T^{\prime}\right)$. Since $G^{\prime}-E\left(T^{\prime}\right)$ is a star forest, at least one of $x$ and $y$ has degree 1 in $G^{\prime}-E\left(T^{\prime}\right)$, say $x$. Now $T=T^{\prime}+u y$ is a starlit $v$-full spanning tree in $G$.

Let $C$ be a cycle in $H$ for which the component of $G-E(C)$ containing $v$ has maximal size. Note that $C$ is induced. Let $K$ denote the component of $G-E(C)$ containing $v$.

Claim 6: $H \subseteq K \cup C$.

Proof. Since $C$ is chordless, it suffices to show $V(H) \subseteq V(K \cup C)$. Suppose $u$ is a vertex in $H$ which is not in $K$ or $C$. Let $L$ denote the component of $G-E(C)$ containing $u$. There exists no cycle in $L \cap H$ since that cycle would contradict the choice of $C$. Claim 4 now implies that $L$ contains a path $P$ joining two vertices $a$ and $b$ on $C$ such that all intermediate vertices are in $V(H) \backslash V(C)$. Let $P_{1}$ and $P_{2}$ be the two edge-disjoint subpaths of $C$ joining $a$ and $b$. We may assume that $P_{2}$ contains a vertex of $K$. Now the cycle formed by the union of $P$ and $P_{1}$ contradicts the choice of $C$.

Since $G$ is fragile, the graph $G-E(C)$ is disconnected so there is a vertex $u$ on $C$ which is not in $K$. Since $C$ is induced, the vertex $u$ has exactly two neighbours on $C$. Claim 6 implies that all neighbours of $u$ not on $C$ have degree 2. Now $d_{H}(u)=2$ and $u \neq v$. By Claim 5, we have $d_{G}(v)=2$ and $u v \in E(G)$, which implies that $u$ is in $K$, contradicting our choice of $u$.

\section{References}

[1] F. Abdolhosseini, S. Akbari, H. Hashemi, and M. Moradian, HoffmannOstenhof's conjecture for traceable cubic graphs, arXiv:1607.04768, (2016).

[2] S. Akbari, T. R. Jensen, And M. Siggers, Decompositions of graphs into trees, forests, and regular subgraphs, Discrete Mathematics, 338 (2015), pp. 1322 - 1327.

[3] M. O. Albertson, D. M. Berman, J. P. Hutchinson, and C. Thomassen, Graphs with homeomorphically irreducible spanning trees, Journal of Graph Theory, 14 (1990), pp. 247-258. 
[4] P. J. Cameron, Research problems from the BCC22, Discrete Mathematics, 311 (2011), pp. $1074-1083$.

[5] R. J. Douglas, NP-completeness and degree restricted spanning trees, Discrete Mathematics, 105 (1992), pp. $41-47$.

[6] A. Hoffmann-Ostenhof, Nowhere-zero flows and structures in cubic graphs, $\mathrm{PhD}$ thesis, University of Vienna, 2011.

[7] A. Hoffmann-Ostenhof, T. Kaiser, and K. Ozeki, Decomposing planar cubic graphs, Journal of Graph Theory, 88 (2018), pp. 613-640.

[8] R. LI, AND Q. CuI, Spanning trees in subcubic graphs, Ars Combinatoria, 117 (2014), pp. 411-415.

[9] J. Malkevitch, Spanning trees in polytopal graphs, Annals of the New York Academy of Sciences, 319 (1979), pp. 362-367.

[10] K. Ozeki And D. Ye, Decomposing plane cubic graphs, European Journal of Combinatorics, 52 (2016), pp. $40-46$. 